Check for updates

Cite this: J. Mater. Chem. C, 2022, 10,4424

Received 2nd December 2021, Accepted 21st February 2022

DOI: $10.1039 / d 1 t c 05794 d$

rsc.li/materials-c

\section{Cross-conjugated isothianaphthene quinoids: a versatile strategy for controlling electronic structures $\dagger$}

\author{
Keitaro Yamamoto, ${ }^{\text {ab }}$ Sergio Moles Quintero, ${ }^{c}$ Seihou Jinnai, (D) a Eunjeong Jeong, ${ }^{d}$ \\ Kyohei Matsuo, (iD d Mitsuharu Suzuki, ${ }^{e}$ Hiroko Yamada, (iD d Juan Casado (iD *c and \\ Yutaka le (iD *af
}

\begin{abstract}
The elucidation of new structure-property relationships in $\pi$-conjugated molecules bearing quinoidal moieties is of relevance because of their use in organic electronics applications and their traditional assimilation as models of doped conducting polymers. Quinoidal oligothiophenes are ground state electronic hybrids between closed-shell Kekulé quinoidal and open-shell aromatic diradicaloid forms. The prominent contribution of the diradical character in longer oligomers beyond thiophene 4-mers results in a low stability, thereby limiting the ability to tune their properties. Thus, the control of these quinoidal/aromatic contributions is an important prerequisite to develop long quinoidal oligothiophenes. To address this problem, a series of quinoidal pentathiophenes with benzene-annelated isothianaphthene units were designed and successfully synthesized as stable structures. Combined molecular spectroscopies and theoretical modelling indicated that cross-conjugation appears upon the introduction of multiple benzene-annelated units, and that the number and position of the benzeneannelated units have a significant influence on the quinoidal/aromatic/cross-conjugated electronic structures. The newly developed quinoidal pentathiophenes functioned as organic semiconducting materials in transistor and near infrared phototransistor devices. This study demonstrates that modification of the cross-conjugated quinoidal structure is a promising strategy for fine-tuning electronic structures in $\pi$-extended quinoidal systems, which could help us to understand unique $\pi$-electronic features and to develop novel organic electronic materials.
\end{abstract}

\section{Introduction}

Considerable progress has been achieved in the development of $\pi$-conjugated oligomers and polymers for possible applications

\footnotetext{
${ }^{a}$ The Institute of Scientific and Industrial Research (SANKEN), Osaka University, 8-1 Mihogaoka, Ibaraki, Osaka 567-0047, Japan.

E-mail: yutakaie@sanken.osaka-u.ac.jp

${ }^{b}$ Tokyo Metropolitan Industrial Technology Research Institute, 2-4-10 Aomi Koto-ku, Tokyo 135-0064, Japan

${ }^{c}$ Department of Physical Chemistry, University of Malaga, Campus de Teatinos $s / n$, 29071 Malaga, Spain

${ }^{d}$ Division of Materials Science, Nara Institute of Science and Technology (NAIST), 8916-5 Takayama-cho, Ikoma, Nara 630-0192, Japan

${ }^{e}$ Division of Applied Chemistry, Graduate School of Engineering, Osaka University, 2-1 Yamadaoka, Suita, Osaka 565-0871, Japan

${ }^{f}$ Innovative Catalysis Science Division, Institute for Open and Transdisciplinary Research Initiatives (OTRI), Osaka University, 2-1 Yamadaoka, Suita

Osaka 565-0871, Japan

$\dagger$ Electronic supplementary information (ESI) available: TGA, UV-Vis-NIR, Raman, NICS, XRD, AFM, CV, FET, OPT, PESA results, synthetic detail, NMR charts, and calculated details. See DOI: 10.1039/d1tc05794d
}

to organic semiconducting materials in organic electronics such as organic light-emitting diodes, organic field-effect transistors (OFETs), and organic solar cells (OSCs). ${ }^{1-3}$ Recently, growing efforts have been devoted to the development of $\pi$-conjugated systems with extended $\pi$-conjugation to acquire narrow energy gaps and red-shifted absorption, which has enabled the application of such systems in new electronic devices, such as near infrared (NIR)-type OSCs, photodetectors, and visible transparent electrodes. ${ }^{4,5}$ It has been documented that the carrier transport characteristics of such $\pi$-conjugated systems correlate with the formation of doped states of quinoidal structures. ${ }^{6}$ In this context, structurally well-defined quinoidal molecules represented by quinoidal oligothiophenes have been extensively investigated not only to understand their distinctive properties, but also to investigate their application to organic semiconducting materials. ${ }^{7-12} \mathrm{~A}$ unique feature of the electronic structures of quinoidal oligothiophenes includes the description of their ground electronic state as a competition between two resonant forms: closed-shell Kekulé quinoidal and open-shell aromatic diradicaloid forms ${ }^{13}$ (Scheme 1(a)). ${ }^{14-16}$ 
(a)

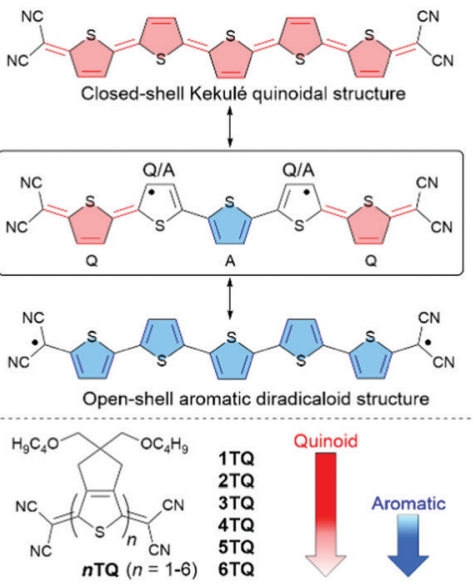

(b)

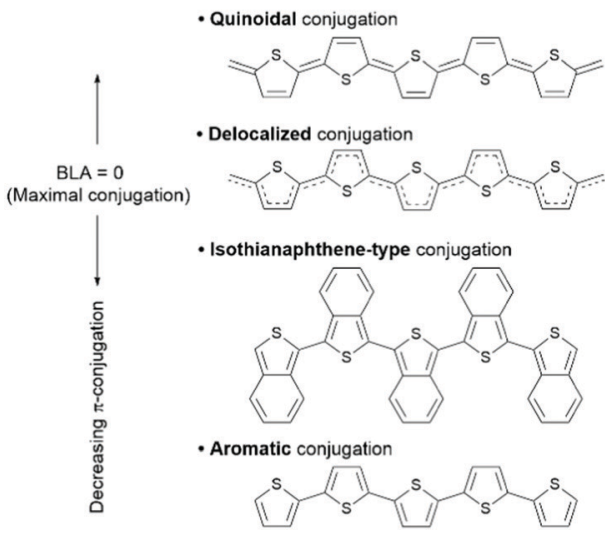

Unexplored region

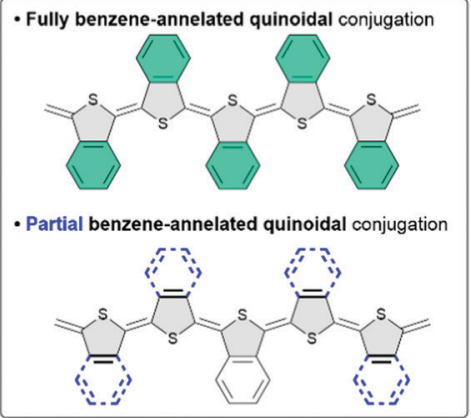

Scheme 1 (a) Closed-shell and open-shell diradical resonance in quinoidal pentathiophene with the balanced structure in the square highlighting the mixture of aromatic (A), quinoidal $(Q)$, and intermediate $(Q / A)$ characters from ref. 15. (b) $\pi$-Conjugation evolution as a function of the bond length alternation (BLA) pattern among families of aromatic and quinoidal oligothiophenes and isothianaphthene aromatic oligothiophenes that highlights the unexplored region of benzene-annelated quinoidal oligothiophenes. Benzene rings in blue color depict the different extent of benzene-annelation in quinoidal pentathiophenes under study.

One co-author of the current article, Casado, revealed that short quinoidal oligothiophenes such as 2 TQ and 3 TQ dominantly contribute to the closed-shell quinoidal structure, while the diradical character becomes prominent upon increasing the number of thiophene rings for longer quinoidal oligothiophenes beyond thiophene 4-mer. ${ }^{15,16}$ This increased diradical character results in poorly stable quinoidal molecules. In fact, the precedent of quinoidal thiophene 5 -mer is still limited to $\mathbf{5 T Q},{ }^{17}$ which hampers the fine-tuning of their molecular properties. Therefore, control of their quinoidal/aromatic electronic structures is of particular interest for the development of long quinoidal oligothiophenes with tuned electronic functions.

The incorporation of the benzo[c]thiophene (isothianaphthene) unit into the polythiophene framework is a pioneering approach for increasing the quinoidal character and $\pi$-electron delocalization that exploits strengthening of the $\mathrm{C}-\mathrm{C}$ single bond at the $\beta$ position of the thiophene ring upon benzene-annelation. ${ }^{18}$ In Scheme 1(b), we summarize the scale of the $\pi$-conjugation degrees achieved in thiophene-based aromatic and quinoidal systems on which we place the isothianaphthene-based molecules between these two because of the mentioned quinoidization effect upon benzeneannelation of aromatic cores. The lacking family in this scheme is that of benzene-annelated quinoidal oligomers. A scientific interest in these compounds is the potential modulation of the diradical character in long quinoidal oligothiophenes caused by the inclusion of isothianaphthene unit. We have achieved some progress in this direction; ${ }^{19,20}$ we recently developed a quinoidal thiophene 3-mer bearing fully benzene-annelation; however, the effect of benzene-annelation on the electronic properties was found to be modest, since benzene-annelation accentuated the preexisting quinoidal electronic structure. ${ }^{20}$ This result indicates that the scenario in longer quinoidal oligothiophenes upon benzeneannelation is completely different and challenging because pristine quinoidal molecules have mixed quinoidal/aromatic electronic structures attributed to the prominent contribution of the diradical character. For example, 5TQ has a central ring with an aromatic structure, while the two terminal rings are quinoidal with aromaticquinoidal transition for the two in between (Scheme 1(a)). ${ }^{15,16}$ This phenomenon inspired us to consider that the partial benzeneannelation of the rings and positional isomerization in addition to full benzene-annelation are attractive in the context of molecular design for precisely controlling quinoidal/aromatic electronic structures.

In this study, we designed and conducted a full structural and electronic characterization of new bis(dicyanomethylene)substituted quinoidal pentathiophenes with partial, positional isomerism and total benzene-annelation of their thiophene rings. These are 5TQ-B5 with full thiophene annelation and two different isomers (5TQ-B3 and 5TQ-BBB) with partial and positional isomerization three-fold annelation and a symmetric mono-annelated pentamer 5TQ-B (Fig. 1). A close comparison was performed with non-annelated quinoidal and aromatic

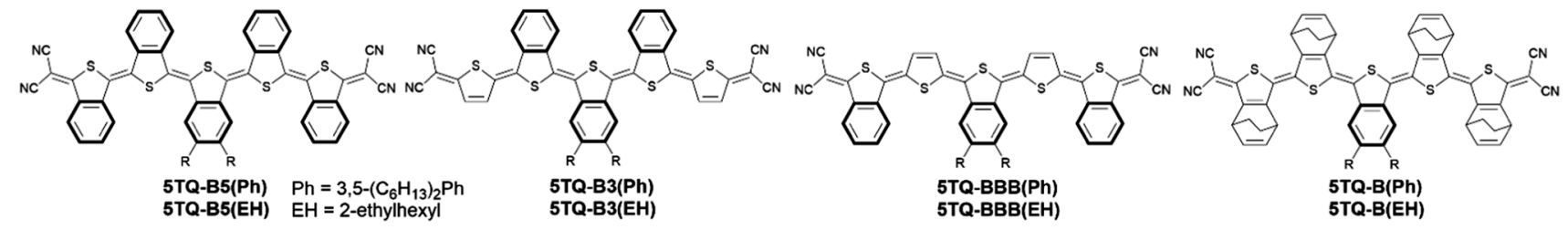

Fig. 1 Chemical structures of the benzene-annelated quinoidal pentathiophenes studied in this work. 
oligothiophene derivatives; a selection of these were implemented as semiconducting materials in OFETs, and in the case of 5TQB5(EH), as NIR photosensitizers in organic phototransistors (OPTs).

\section{Results and discussion}

\section{Synthesis}

Synthetic details to prepare target compounds are summarized in Scheme 2. Based on the contribution of the ortho-quinoid electronic structure, benzo[c]thiophene is known as an unstable scaffold. Except for electrochemical polymerization, ${ }^{18 a, c}$ the $\pi$-extension of the isothianaphthene-repeated oligomer is limited up to thiophene 3-mer; these molecules possess end-capping with electron-withdrawing substituents to ensure chemical stability. ${ }^{21}$ Hence, we plan to synthesize 4,7-dihydro-4,7ethanobenzo[ $c]$ thiophene-based oligomers and utilize a thermal retro Diels-Alder reaction in the final step of the synthetic route to prevent the transformation of benzo[c]thiophene-based thiophene 5-mers. ${ }^{21}$ Diiodo compound 1a was prepared according to our previous report. ${ }^{20}$ The Suzuki-coupling reaction of $\mathbf{1 a}$ and the boronic ester 2 afforded the $\pi$-conjugated framework of thiophene 5-mer; this reacted with $\mathrm{N}$-iodosuccinimide (NIS) to provide compound $\mathbf{3 a}$. The cross-coupling reaction of $\mathbf{3 a}$ with sodium dicyanomethanide, followed by oxidation using 2,3dichloro-5,6-dicyano- $p$-benzoquinone (DDQ), afforded the quinoidal pentathiophene $\mathbf{5 T Q - B}(\mathbf{P h})$ in $44 \%$ yield. The same synthetic protocol was adopted for the synthesis of 5TQ-B(EH), $\mathbf{6 a}, \mathbf{6 b}$, 9a, and 9b. Note that all the synthesized quinoidal molecules have enough ambient stability due to the fine-tuned diradical character.

Thermogravimetric analysis (TGA) was conducted to investigate if thermal retro Diels-Alder reactions can be applied to the precursor molecules (5TQ-B(EH), 6b, and 9b). As indicated in Fig. 2, these molecules presented the first weight loss of $c a$.

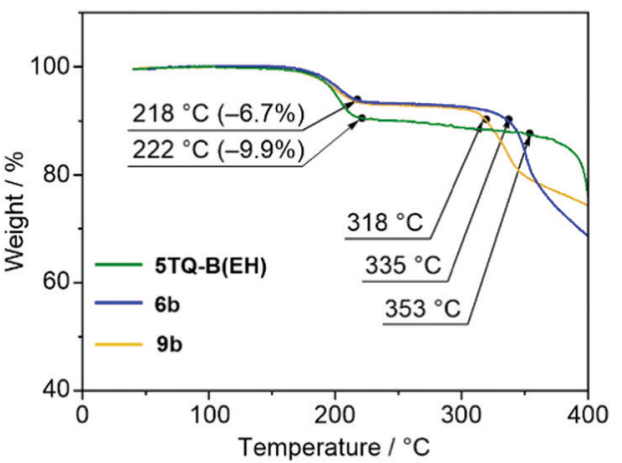

Fig. 2 TGA curves for $\mathbf{5 T Q}-\mathbf{B}(\mathrm{EH})$ (green), $\mathbf{6 b}$ (blue), and $\mathbf{9 b}$ (yellow) with a heating rate of $10{ }^{\circ} \mathrm{C} \mathrm{min}^{-1}$ in an $\mathrm{N}_{2}$ atmosphere.

$6.7 \%$ and $9.9 \%$ in the temperature range of $190-220{ }^{\circ} \mathrm{C}$ because of eliminating ethylene molecules. These results suggest that 5TQ-B(EH), 6b, and 9b were thermally converted to 5TQB5(EH), 5TQ-B3(EH), and 5TQ-BBB(EH). The TGA curves of the second weight loss correspond to the decomposition of 5TQ-B5(EH), 5TQ-B3(EH), and 5TQ-BBB(EH). The decomposition temperature was increased in the order of 5TQ-BBB(EH) $\left(318^{\circ} \mathrm{C}\right)$, 5TQ-B3(EH) $\left(335{ }^{\circ} \mathrm{C}\right)$, and 5TQ-B5(EH) $\left(353{ }^{\circ} \mathrm{C}\right)$. The TGA measurements of 5TQ-B(Ph), 6a, and 9a demonstrated similar behaviors (Fig. S1 in the ESI $\dagger$ ), which indicates that the replacement of 2-ethylhexyl groups with 3,5-dihexylbenzene groups had little influence on the thermal properties of the molecules. These results suggest that the thermal conversion reactions of the synthesized precursors were performed at $260{ }^{\circ} \mathrm{C}$ under vacuum conditions to yield targets 5TQ-B5(EH), 5TQ-B3(EH), 5TQ-BBB(EH), 5TQ-B5(Ph), 5TQ-B3(Ph), and 5TQ-BBB(Ph). The detailed synthetic methods and characterization data are summarized in the ESI. $\dagger$ 5TQ-B5(EH), 5TQ-B3(EH), and 5TQ-BBB(EH) showed very poor solubility, and moderate improvement
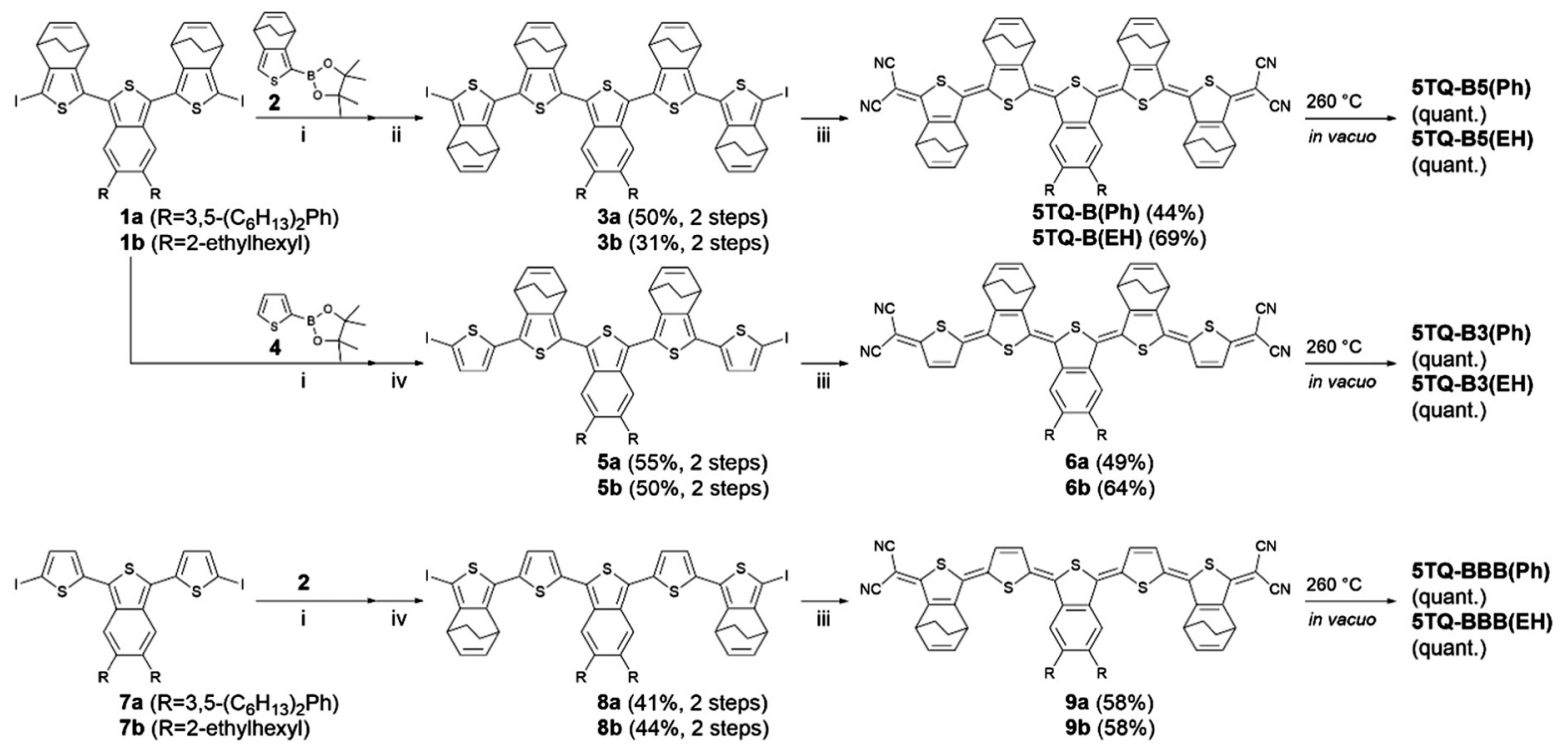

Scheme 2 Synthesis of quinoidal pentathiophenes. Reaction conditions: (i) $\mathrm{Pd}_{2}(\mathrm{dba})_{3} \cdot \mathrm{CHCl}_{3}, \mathrm{SPhOs}_{3} \mathrm{~K}_{3} \mathrm{PO}_{4}, \mathrm{THF} / \mathrm{H}_{2} \mathrm{O}, 65^{\circ} \mathrm{C}$; (ii) $\mathrm{NIS}, \mathrm{DMF} / \mathrm{CHCl}, 0{ }^{\circ} \mathrm{C}$ to room temperature; (iii) $\mathrm{NaH}$, malononitrile, $\mathrm{Pd}\left(\mathrm{PPh}_{3}\right)_{4}, \mathrm{dppf}, \mathrm{THF}, 75^{\circ} \mathrm{C}$; DDQ, $\mathrm{HCl} / \mathrm{CHCl}_{3}, \mathrm{O}^{\circ} \mathrm{C}$; (iv) $\mathrm{LDA}, \mathrm{THF},-78^{\circ} \mathrm{C}$ to room temperature; $\mathrm{I}_{2},-78{ }^{\circ} \mathrm{C}$. 
(a)

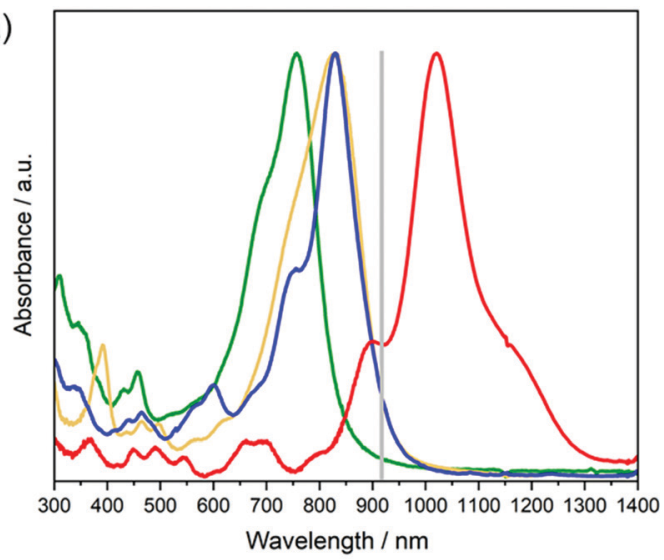

(b)

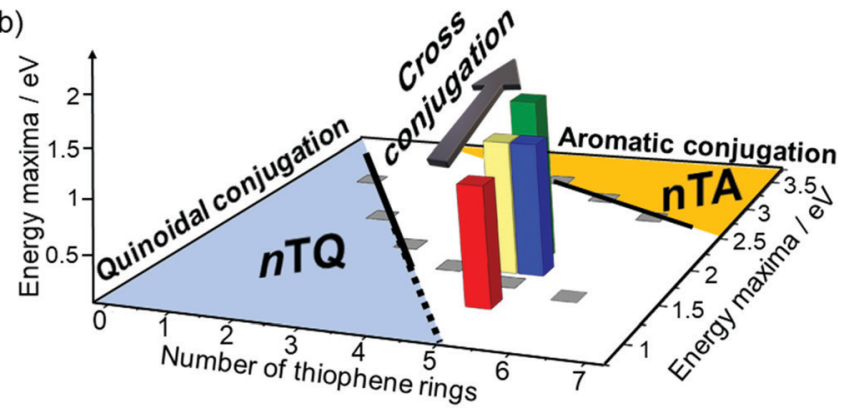

Fig. 3 (a) UV-Vis-NIR absorption spectra of 5TQ-B5(Ph) (green), 5TQB3(Ph) (blue), 5TQ-BBB(Ph) (yellow), and 5TQ-B(Ph) (red) in TCE. Gray line indicates the $\lambda_{\max }$ of $\mathbf{5 T Q}$. (b) Representation of the evolution of the energy peak maxima of the lowest energy band of absorption spectra of $\boldsymbol{n T Q} \mathbf{Q}^{16}$ and $n T^{22}$ compounds from the monomer to the hexamer as representative cases both of linear conjugations. In the vertical axis is shown the evolution of the energy maxima of the absorption bands in the series of benzeneannelated quinoidal pentamers which on increasing the number of benzene-annelating units cross-conjugation increases starting in 5TQ$\mathbf{B}(\mathbf{P h})$ (red bar), 5TQ-B3(Ph) (blue bar), 5TQ-BBB(Ph) (yellow bar), and 5TQ-B5(Ph) (green bar). Cross-conjugation evolution in the vertical axis is thus out of the plane of linear aromatic and quinoidal conjugations.

$\left(<1 \mathrm{mg} \mathrm{mL}^{-1}\right.$ at $\left.25{ }^{\circ} \mathrm{C}\right)$ in 5TQ-B5(Ph), 5TQ-B3(Ph), and 5TQ$\mathbf{B B B}(\mathbf{P h})$ in organic solvents such as chloroform and 1,1,2,2tetrachloroethane (TCE), which allows physical measurements in the solution.

\section{UV-Vis-NIR electronic absorption spectra}

The UV-Vis-NIR absorption spectra of 5TQ-B5(Ph), 5TQ-B3(Ph), 5TQ-BBB(Ph), and 5TQ-B $(\mathbf{P h})$ in TCE solutions are presented in Fig. 3(a), and the photophysical data are summarized in Table 1 . The absorption maximum $\left(\lambda_{\max }\right)$ of $\mathbf{5 T Q} \mathbf{T} \mathbf{B}(\mathbf{P h})$ is observed in the near-infrared region at $\lambda_{\max }=1021 \mathrm{~nm}$, redshifted with $\lambda_{\max }=913 \mathrm{~nm}$ in 5TQ. Compared to 5TQ-B(Ph), 5TQ-B5(Ph) showed a remarkable blue-shift absorption with $\lambda_{\max }$ at $757 \mathrm{~nm}$. In addition, the $\lambda_{\max }$ values for 5TQ-B3(Ph) and 5TQ-BBB(Ph) were located close to $830 \mathrm{~nm}$ between those of 5TQ-B5(Ph) and 5TQ-B(Ph). Fig. 3(b) compares the chain length dependence of the energies of the band maxima of the present compounds compared to those of the non-annelated quinoidal thiophene analogues $(\boldsymbol{n T Q}){ }^{16}{ }^{16}$ and of the most representative aromatic oligothiophene analogues $(\boldsymbol{n T A}) .{ }^{22}$ In the $n$ TQ series from the monomer to the trimer, the quinoidal contribution is $100 \%$, based on which the extrapolation of this behavior (see dotted lines in Fig. 3(b)) for longer compounds discloses their expected band energy maxima (i.e., it would be entirely quinoidal). The same is shown for the aromatic oligothiophene $n$ TA behavior in Fig. 3(b). Between these two extreme quinoidal/ aromatic behaviors (highlighted as black solid lines), oligothiophenes with mixed quinoidal/aromatic electronic composition are recognized in the graph as transiting from one regime to another; this occurs incipiently for 4TQ and 5TQ and 6TQ because of the progressive aromatization of the quinoidal core attributed to the stabilization of the open-shell diradical aromatic structure. ${ }^{16}$ In this quinoidal-aromatic map/plane in Fig. 3(b), $\mathbf{5 T Q}-\mathbf{B}(\mathbf{P h})$ is placed closer to the quinoidal line than 5TQ, which reveals a greater quinoidal character in the latter. According to the progression on 5TQ $\rightarrow$ 5TQ-B $(\mathbf{P h})$ upon benzene-annelation, 5TQ-B3(Ph) with three benzene-annelated thiophenes are expected to gain more quinoidal character; however, the energy peak maxima, which is unlike 5TQ $\rightarrow$ $\mathbf{5 T Q}-\mathbf{B}(\mathbf{P h})$, separate from the quinoidal area; this behavior is more marked in 5TQ-B5(Ph). This evolution on 5TQ-B(Ph) $\rightarrow$ 5TQ-B3(Ph) $\rightarrow$ 5TQ-B5(Ph) is not observed as a progressive gain of aromatic character (such as on $4 \mathrm{TQ} \rightarrow 5 \mathrm{TQ} \rightarrow 6 \mathrm{TQ}$ ); instead, it emerges from the effect of cross-conjugation. ${ }^{23}$ Crossconjugated $\pi$-electronic paths produce blue shifts of the absorption peak maxima (opening the optical energy gap) compared to homologues with linear $\pi$-conjugation. Hence, the studied benzene-annelated compounds cannot be represented by a simple quinoidal-aromatic competition, but a third crossconjugation factor by benzene-annelation enters into play. This cross-conjugation might impart electron wavefunction draining from the linear-conjugated path, and it has a significant impact on the electronic, optical, and molecular properties.

\section{Solid-state Raman spectra}

The $1064 \mathrm{~nm}$ FT-Raman spectra in the solid state of 5TQ-B(Ph) compared with that of 5TQ are shown in Fig. 4(a). The Raman

Table 1 Photophysical and electrochemical properties of molecules

\begin{tabular}{|c|c|c|c|c|c|c|c|}
\hline Compounds & $\lambda_{\max }{ }^{a} / \mathrm{nm}$ & $\Delta E_{\mathrm{g}}^{\mathrm{opt} a} / \mathrm{eV}$ & $E^{\mathrm{oxb}} / \mathrm{V}$ & $E^{\mathrm{red} b} / \mathrm{V}$ & $E_{\text {Hомо }}^{c} / \mathrm{eV}$ & $E_{\mathrm{LUMO}}{ }^{d} / \mathrm{eV}$ & $\Delta E_{\mathrm{g}}^{\mathrm{DPV} e} / \mathrm{eV}$ \\
\hline 5TQ-B5(Ph) & 757 & 1.48 & 0.30 & -1.06 & -5.10 & -3.74 & 1.36 \\
\hline 5TQ-B3(Ph) & 829 & 1.34 & 0.35 & -0.76 & -5.15 & -4.04 & 1.11 \\
\hline 5TQ-BBB(Ph) & 830 & 1.34 & 0.21 & -0.81 & -5.01 & -3.99 & 1.02 \\
\hline 5TQ-B(Ph) & 1021 & 0.95 & n.d. & n.d. & n.d. & n.d. & n.d. \\
\hline
\end{tabular}


(a)

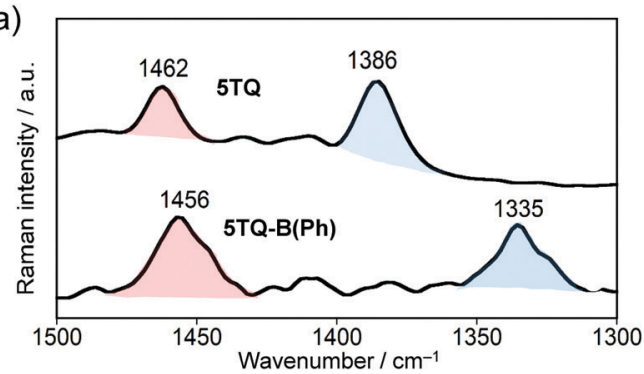

(b)

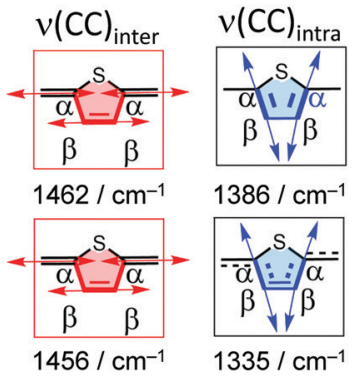

(c)
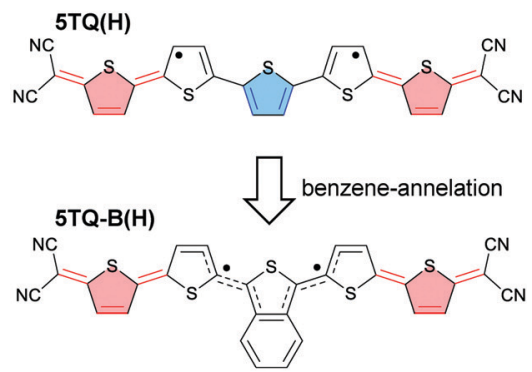

Fig. 4 (a) FT-Raman spectra recorded with the $1064 \mathrm{~nm}$ excitation in solid state at room temperature of $\mathbf{5 T Q}-\mathbf{B}(\mathbf{P h})$ and $\mathbf{5 T Q}$. (b) Vibrational dynamics of the main Raman bands associated with the CC stretching modes of the quinoidal (red) and aromatic aromatic (blue) rings. (c) Structural evolution of the quinoidal rings upon benzene-annelation.

spectrum of 5TQ displays two main bands of similar intensity at 1462 and $1386 \mathrm{~cm}^{-1}$, which are associated with the CC bond stretching modes placed at rather different places of the conjugated skeleton; that at $1462 \mathrm{~cm}^{-1}$ is attributed to the CC inter-ring bond stretching modes [i.e., $\nu(\mathrm{CC})_{\text {inter }}$ in Fig. 4(b)] with the main contribution from the CC bond connecting to the dicyano groups; that at $1386 \mathrm{~cm}^{-1}$ belongs to the CC stretching modes of the two intra-ring $\alpha-\beta$ CC bonds of the thiophene [i.e., $\nu(\mathrm{CC})_{\text {intra }}$ in Fig. 4(b)]. The $1386 \mathrm{~cm}^{-1}$ wavenumber of the $\nu(\mathrm{CC})_{\text {intra }}$ Raman band in 5TQ is associated with the aromatization of the central three rings (more marked in the central ring) because of the formation of a diradical structure in 5TQ, as depicted in Scheme 1(a) and Fig. 4(c). This description of 5TQ with a central aromatic part and the outermost thiophenes with more quinoidal character is pivotal for understanding the role of benzene annelation. The Raman spectrum of $\mathbf{5 T Q - B}(\mathbf{P h})$ is similar to that of 5TQ but with wavenumber downshifts of the two CC stretching bands, in particular the $1386 \rightarrow 1335 \mathrm{~cm}^{-1}$ for the $\nu(\mathrm{CC})_{\text {intra }}$ band. This indicates a weakening of the $\alpha-\beta$ bonds consistent with a decrease in the overall aromatic character of the thienyl rings, or conversely, a gain of quinoidal character (see Fig. 4(c)), as also revealed in the UV-Vis-NIR discussion.

The FT-Raman spectra of the benzene-annelated quinoidal pentathiophenes are shown in Fig. 5(a). From 5TQ-B(Ph) to 5TQ-B5(Ph), the two discussed Raman band patterns disappear, and one main Raman band emerges at $1468 \mathrm{~cm}^{-1}$. The theoretical Raman spectrum of 5TQ-B5(H) is calculated and shown in Fig. 5(b), which reveals a good resemblance with the experimental spectrum with a very strong theoretical Raman band at $1457 \mathrm{~cm}^{-1}$, this correlates with the experimental results at $1468 \mathrm{~cm}^{-1}$. According to a theoretical vibrational eigenvector, this $1457 \mathrm{~cm}^{-1}$ band can be described as the $\nu(\mathrm{CC})_{\text {inter }}$ mentioned above; It indicates that the full benzene annelation of the quinoidal pentathiophene core changes the vibrational dynamics of the $\pi$-conjugated system, and it results in the disappearance of the $\nu(\mathrm{CC})_{\text {intra }}$. Raman band of 5TQ and 5TQ-B(Ph). This can be understood by considering that benzeneannelation/cross-conjugation breaks the $\pi$-electron delocalization and transmission through the intra-ring $\mathrm{CC}$ bonds, which results in less efficient linear $\pi$-conjugation that weakens the $\nu(\mathbf{C C})_{\text {intra }}$ Raman intensity to the point of clearance (Fig. 5(c)). In 5TQ-

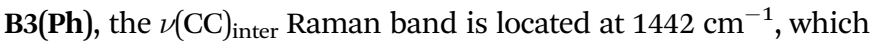
agrees with an intermediate scenario between 5 TQ-B(Ph) and 5TQ-B5(Ph). In 5TQ-BBB(Ph), the main band at $1404 \mathrm{~cm}^{-1}$ between the wavenumbers of $\mathbf{5 T Q - B}(\mathbf{P h})$ at $1335 \mathrm{~cm}^{-1}$ and 5TQB3(Ph) at $1442 \mathrm{~cm}^{-1}$ was measured, as accompanied by another medium-large intensity Raman band at $1420 \mathrm{~cm}^{-1}$ that comprises the two-band pattern featured by $\mathbf{5 T Q - B}(\mathbf{P h})$ and 5TQ; it indicates that the alternant mode of non-annelated and annelated thiophenes produces a smaller interference in the linear conjugation path by cross-conjugation than that in the isomeric 5TQ-B3(Ph). (a)

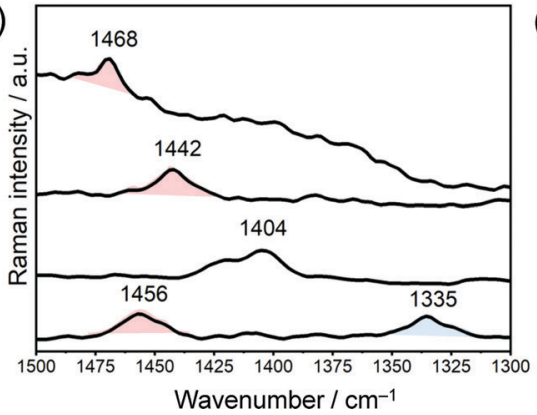

(b)

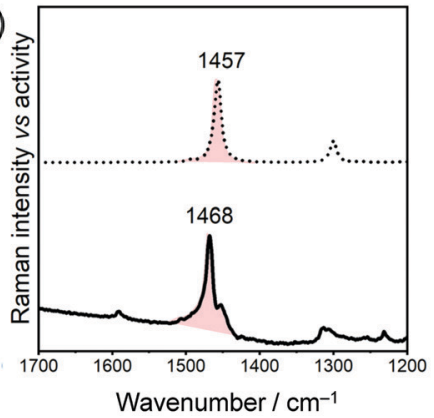

(c)

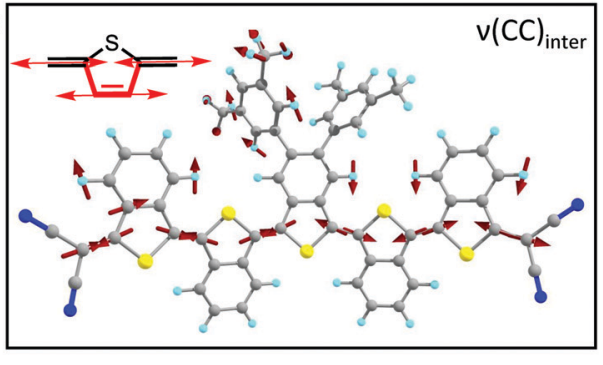

Vibrational eigenvector

Fig. 5 (a) $1064 \mathrm{~nm}$ FT-Raman spectra of benzene-annelated quinoidal pentathiophenes in the solid state at room temperature from the bottom: 5TQ-B(Ph), 5TQ-BBB(Ph), 5TQ-B3(Ph), and 5TQ-B5(Ph). (b) Comparison of B3LYP/6-31G(d,p) theoretical Raman spectrum of a model compound of 5TQ-B5(H) (top) compared with the experimental Raman spectrum of 5TQ-B5(Ph) (bottom). (c) B3LYP/6-31G(d,p) vibrational eigenvector of the $1457 \mathrm{~cm}^{-1}$ Raman band of the theoretical spectrum of $\mathbf{5 T Q}-\mathbf{B 5}(\mathbf{H})$. The inset is the comparison with the previously described $\nu(C C)_{\text {inter }}$. 
The multiple connectivity bonding pattern on the $\beta$ carbons of thiophenes in the presence of benzenes originates from the emergence of cross-conjugation. However, we wonder why single benzene-annelation 5TQ-B(Ph) strengthens quinoidization but not cross-conjugation, which is operative in compounds with multiple benzene annelations. Benzene annelation in the central ring of 5TQ-B(Ph) acts on an aromatic ring (see 5TQ in Scheme 1(a) and Fig. 4(c)), whereas in the case of multiannelation, benzenes are fused with thiophenes that are mostly quinoidal (Scheme 1(a) and Fig. 4(c) for the distribution of aromatic and quinoidal rings in 5TQ) in 5TQ-BBB(Ph) in 5TQ-B3(Ph) and fully quinoidal in 5TQ-B5(Ph). Thus, the spectroscopic behavior in 5TQ-B(Ph) reveals that the effect of the electronic structure of the mono-benzene-annelation of 5TQ is identical to that of the benzene-annelation of aromatic oligothiophenes (i.e., in the case of isothianaphthene aromatic oligomers) in which benzeneannelation produces the quinoidization of aromatic thiophenes. Conversely, multiple benzene-annelation units of quinoidal rings of 5TQ in 5TQ-B3(Ph), 5TQ-BBB(Ph), and 5TQ-B5(Ph) produces cross-conjugation.

\section{Quantum chemical calculations}

Density functional theory (DFT) calculations were performed at the UB3LYP/6-31G(d,p) level (Fig. 6(a) and Fig. S2, ESI $\dagger$ ) to gain insights into the effects of benzene-annelation on the electronic structures and frontier orbital energies. All solubilizing groups were replaced with hydrogen atoms to reduce the computational work. The number of benzene-annelation affects the lowest unoccupied molecular orbital (LUMO) energy level (Fig. 7 where the impact of the confluence of ortho-conjugation, benzo-aromatic, and para-quinoid structures arise on benzene-annelation is in the LUMO orbital) by which decreasing the number of benzene annelation leads to a decrease in the highest occupied molecular orbital (HOMO)-LUMO energy gap in line with the experimental optical gap ( $\left.\Delta E_{\mathrm{g}}^{\mathrm{opt}}\right)$ reduction from the onset and peak maxima of the optical absorption spectra as $1.48 \mathrm{eV}$ in 5TQ-B5(Ph), $1.34 \mathrm{eV}$ in 5TQ-B3(Ph) and 5TQ-BBB(Ph), and $0.95 \mathrm{eV}$ in 5TQ-B(Ph).

Quinoidal systems with narrow HOMO-LUMO energy gaps and aromaticity recovery induce diradical contribution in the ground state. ${ }^{24}$ The diradical character $\left(y_{0}\right)$ defined in terms of the occupation number of the lowest unoccupied natural orbital for optimized model structures is calculated by the

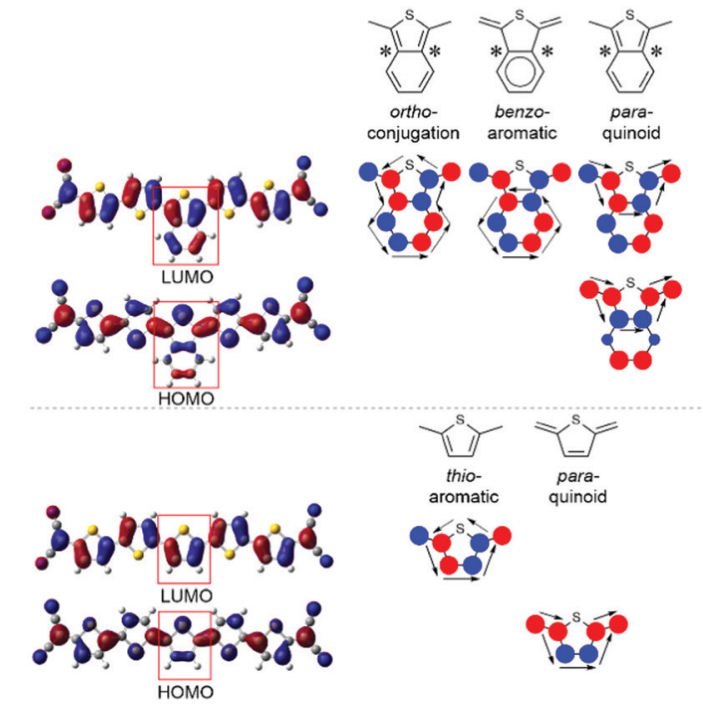

Fig. $7 \mathrm{HOMO}$ and LUMO orbitals of $5 \mathrm{TQ}-\mathbf{B}(\mathrm{H})$ (top) and of $5 \mathrm{TQ}(\mathrm{H})$ (bottom) together with the inter-atomic interactions (arrows) among vicinal atoms in these two orbitals that reveal the presence of wavefunction bifurcation atoms in the benzene-annelated ring of $5 T Q-B(H)$ compared to $\mathbf{5 T Q}(\mathrm{H})$. Valence bond structures equivalent to ortho-conjugation, benzoand thio-aromatic, and para-quinoid are shown for clarity.

spin-unrestricted Hartree-Fock method at the 6-31G(d) level for the studied compounds. ${ }^{25}$ The $y_{0}$ value becomes zero when the $\pi$-system is dominated by a closed-shell quinoid structure, whereas it approaches 1 when increasing the contribution of the open-shell diradical structure. For quinoidal oligothiophenes, $y_{0}$ increases with increasing molecular length because of the increasing number of quinoidal rings, which increases the aromatic stabilization of the diradical form. For instance, $\mathbf{5 T Q}(\mathbf{H})$ discloses $y_{0}=0.73$, and its ground electronic state is featured by a central part (i.e., spreading approximately three central rings, see Scheme 1(a) and Fig. 4(c)) with aromatic-like character flanked by the two radical centers. For $\mathbf{5 T Q - B}(\mathbf{H})$, this amounts to $y_{0}=0.54$, which is smaller than that of $5 \mathbf{T Q}(\mathbf{H})$. This behavior is in good agreement with the removal of the aromatic character in $\mathbf{5 T Q}(\mathbf{H})$ upon benzene-annelation on the central thiophene. The extension of benzene-annelation of quinoidal oligothiophenes to 5TQ-B3(H) and 5TQ-B5(H) provokes the complete closure of the electronic shell with $y_{0}=0.00$, which

(a)

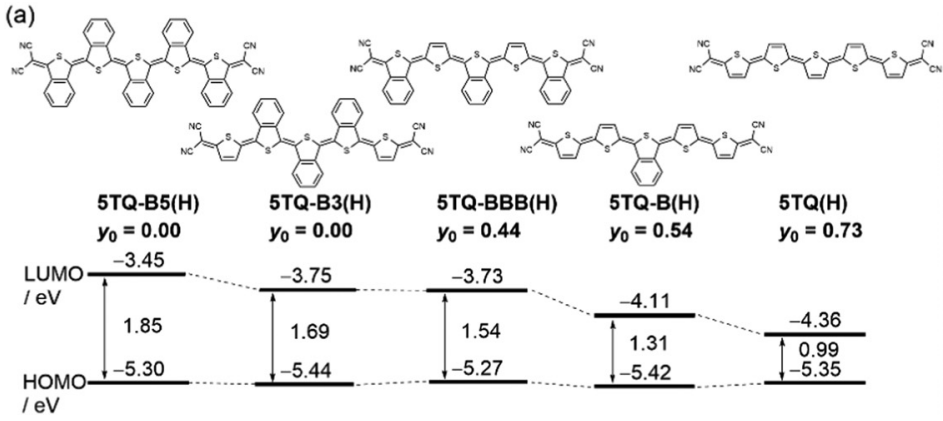

(b)

Fig. 6 (a) Calculated energy levels and $y_{0}$ values and (b) the energy differences between $S_{0}$ and $T_{1}$ of $\mathbf{5 T Q}$-B5(H), 5TQ-B3(H), 5TQ-BBB(H), 5TQ-B(H), and $\mathbf{5 T Q}(\mathrm{H})$. 
agrees with the complete removal of aromatic character upon full thiophene benzene-annelation. On the other hand, 5TQ$\mathbf{B B B}(\mathbf{H})$, which is a positional isomer of 5TQ-B3(H), showed moderate diradical character of $y_{0}=0.44$. This result indicates that the alternant mode of non-annelated and annelated thiophenes leads to increasing the radical character. This trend is in good agreement with the discussions based on 5TQ-BBB(Ph) and 5TQ-B3(Ph) in the Raman spectroscopy measurements. Finally, the energy differences between $S_{0}$ and $T_{1}$ of 5TQ-B5(H), 5TQ-B3(H), 5TQ-BBB(H), 5TQ-B(H), and 5TQ(H) were calculated to be $21.9,17.1,11.3,4.8$, and $2.7 \mathrm{kcal} \mathrm{mol}^{-1}$, respectively, in line with the previous discussion of $y_{0}$ (Fig. 6(b)).

$\operatorname{NICS}(1.7)_{z z}$ values are obtained for the benzene and thiophene rings of the studied compounds and are summarized in Table S1 $(\mathrm{ESI} \dagger)$. For the central benzene rings, the NICS values are more negative for 5TQ-B(H) $\rightarrow$ 5TQ-BBB(H) $\rightarrow$ 5TQ-B3(H) $\rightarrow$ 5TQB5(H), which is consistent with the gain of aromatic character with increasing cross-conjugation. This produces electronic isolation between the benzene and the linear-conjugated path. This is accompanied by a decrease in the aromatic character of the fused thiophene, and it is similar to that of benzene in 5TQ$\mathbf{B}(\mathbf{H})$ and it progressively diminishes its aromatic character, eventually becoming nonaromatic in 5TQ-B5(H).

As shown in Fig. S3 (ESI $\dagger$ ), the ${ }^{1} \mathrm{H}$ NMR spectra of 5TQ-B5(Ph) and 5TQ-B3(Ph) in a solution of 1,1,2,2-tetrachloroethane- $d_{2}$ exhibited sharp peaks in the aromatic region even at $130{ }^{\circ} \mathrm{C}$, which indicated their closed-shell electronic structure. In contrast, the ${ }^{1} \mathrm{H}$ NMR spectrum of 5 TQ-BBB(Ph) showed broadened signals for the protons of the thiophene rings, which is explained by the thermal population of the excited triplet species. In the case of 5TQ-B(Ph), the ${ }^{1} \mathrm{H}$ NMR spectrum in the solution exhibited broadened signals, besides those for phenyl substituents, even at $25{ }^{\circ} \mathrm{C}$, which indicates the triplet state is dominant for 5TQ-B(Ph).

\section{Electrochemical measurements}

The influence of the position and number of benzeneannelations on redox behavior is determined via differential pulse voltammetry (DPV) in 5TQ-B5(Ph), 5TQ-B3(Ph), and 5TQ-BBB(Ph) in TCE containing $0.1 \mathrm{M}$ tetrabutylammonium hexafluorophosphate $\left(\mathrm{TBAPF}_{6}\right)$ as a supporting electrolyte. Fig. 8(a) shows that these compounds exhibit oxidation $\left(E^{\mathrm{ox}}\right)$ and reduction processes $\left(E^{\mathrm{red}}\right)$ that exchange the same number of electrons, which indicates that they are one electron or singleelectron processes in contrast with $\mathbf{n T Q}$ quinoidal oligothiophenes, which display two-electron reductions. Further, the distinctive behavior of annelated compounds is revealed in the electrochemical potential $E^{\mathrm{ox}}$ and $E^{\mathrm{red}}$ values (Table 1), which are negatively displaced relative to $5 \mathrm{TQ}$ at $-0.16 \mathrm{~V} .{ }^{16}$ In addition, those of 5TQ-B5(Ph) is negatively shifted in comparison with those of 5TQ-B3(Ph), in agreement with the larger deviation of the quinoidal linear $\pi$-conjugation behavior in the cases with more benzenes. Compared to 5TQ-B3(Ph), the alternating introduction of benzene-annelation for $\mathbf{5 T Q - B B B}(\mathbf{P h})$ resulted in slight changes in the reduction potentials. The disruption of the electronic communication in the thienyl chain by
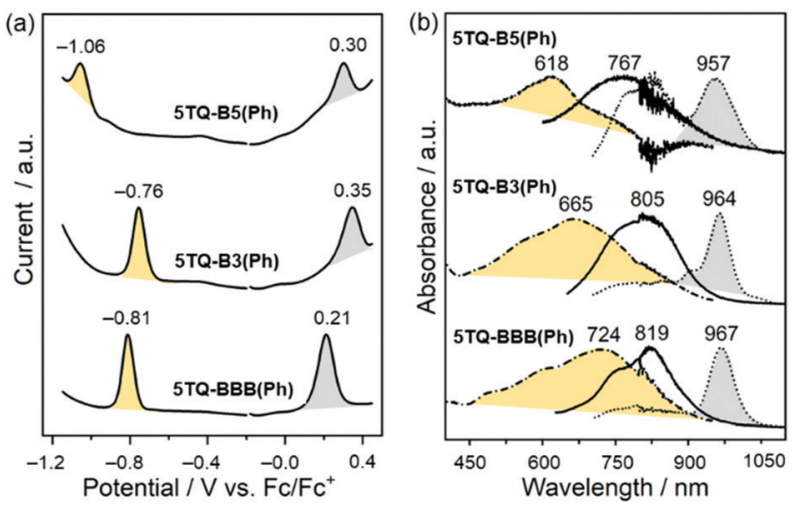

Fig. 8 (a) DPV of 5TQ-B5(Ph), 5TQ-B3(Ph) and 5TQ-BBB(Ph) in TCE containing 0.1 M TBAPF 6 . (b) Spectroelectrochemical UV-Vis-NIR spectra of the reduced (broken lines, yellow shaded) and oxidized (dotted lines, grey shaded) species obtained in the same electrochemical conditions than those use in DPV together with the spectra of the neutrals (solid lines).

cross-conjugation alters the role of the external electron-withdrawing cyano groups, which further affects the oxidation potentials (Table 1). The HOMO and LUMO energy levels of these compounds were estimated from the equations $E_{\mathrm{HOMO}}=-E^{\mathrm{ox}}-4.8$ and $E_{\mathrm{LUMO}}=-E^{\mathrm{red}}-4.8$ presented in Table 1, which follow the same trend as the optical gaps from the absorption spectra.

The UV-Vis-NIR absorption spectra of redox species found in the electrochemical oxidation and reduction processes are obtained by UV-Vis-NIR spectroelectrochemistry; the spectra are shown in Fig. 8(b) (Step-by-step UV-Vis-NIR followed redox processes are shown in Fig. S4, ESI $\dagger$ ). The spectra of the reduced species of the three compounds are always blueshifted compared with the absorption bands of the neutral compounds. In contrast, for the oxidized species, the relevant absorption bands are red-shifted with respect to the neutrals. Interestingly, 5TQ-B3(Ph) and $\mathbf{5 T Q - B B B}(\mathbf{P h})$ display smaller differences between the wavelength maxima of the reduced and oxidized species, which indicates that these two redox species of different charge signs are stabilized in similar ways with moderate or smaller structural changes from one to the other. This is important because 5TQ-B3(Ph) and 5TQ-BBB(Ph) show ambipolar semiconducting behavior, whereas 5TQ-B5(Ph) with larger differences in the optical spectra between the reduced and oxidized species reveals a preferential stabilization of one of the two, which indicates unipolar semiconducting behavior.

\section{Device applications}

We fabricated thin films of 5TQ-B5(EH), 5TQ-B3(EH), and 5TQ$\mathbf{B B B}(\mathbf{E H})$ using the drop-cast method from a hot TCE solution. The X-ray diffraction (XRD) profiles of the drop-cast films of all molecules showed first-order diffraction peaks with $2 \theta=4.8^{-}$ 6.1 $1^{\circ}$, which correspond to the $d$-spacing of 18.4-14.5 $\AA$ (Fig. S5, $\mathrm{ESI} \dagger)$. This length is approximately close to the calculated molecular lengths of quinoidal thiophene 5-mer in the long axis direction ( $c a .20 \AA$ ), and it implies that these molecules are arranged in an edge-on orientation against the $\mathrm{SiO}_{2}$ substrates. 
Table 2 Field-effect transistor characteristics

\begin{tabular}{lllrl}
\hline Compounds & Carrier type & $\mu / \mathrm{cm}^{2} \mathrm{~V}^{-1} \mathrm{~s}^{-1}$ & $V_{\mathrm{th}} / \mathrm{V}$ & $I_{\text {on }} / I_{\text {off }}$ \\
\hline 5TQ-B5(EH) & $\mathrm{p}$ & $6.8 \times 10^{-5}$ & -9 & $10^{3}$ \\
5TQ-B3(EH) & $\mathrm{p}$ & $1.8 \times 10^{-3}$ & -27 & $10^{1}$ \\
& $\mathrm{n}$ & $1.7 \times 10^{-3}$ & 19 & $10^{1}$ \\
5TQ-BBB(EH) & $\mathrm{p}$ & $2.0 \times 10^{-4}$ & -31 & $10^{2}$ \\
& $\mathrm{n}$ & $4.3 \times 10^{-4}$ & 13 & $10^{2}$
\end{tabular}

Atomic force microscopy (AFM) images showed distinct crystalshaped microstructures (Fig. S6, ESI $†$ ). We investigated the charge carrier characteristics using bottom-gate bottomcontact OFET devices. The transfer and output characteristics of these devices are shown in Fig. S7 and S8 (ESI $\dagger$ ); key device parameters such as mobility $(\mu)$, threshold voltage $\left(V_{\text {th }}\right)$, and current on/off ratio $\left(I_{\mathrm{on}} / I_{\text {off }}\right)$ are extracted and summarized in Table 2. 5TQ-B5(EH) demonstrated typical hole-transporting characteristics with a mobility of $10^{-5}$ orders. In contrast, 5TQB3(EH) and 5TQ-BBB(EH) exhibited ambipolar characteristics with well-balanced mobilities of $10^{-3}$ and $10^{-4}$ orders; these values indicate that these compounds possess the appropriate HOMO and LUMO energy levels for hole and electron injection and structural stability of the redox species, as mentioned above. The ambipolar characteristics of 5TQ-B3(EH) and 5TQ$\mathbf{B B B}(\mathbf{E H})$ resulted in low $I_{\mathrm{on}} / I_{\text {off }}$ ratios due to the accumulation of opposite carriers. To examine the stability of these molecules under the biased conditions, the OFETs were repeatedly biased by sweeping the gate voltage. As shown in Fig. S9 (ESI $\dagger$ ), all the devices showed small hysteresis even after 100 cycles. These results indicate that these quinoidal molecules showed enough stability for OFET materials.
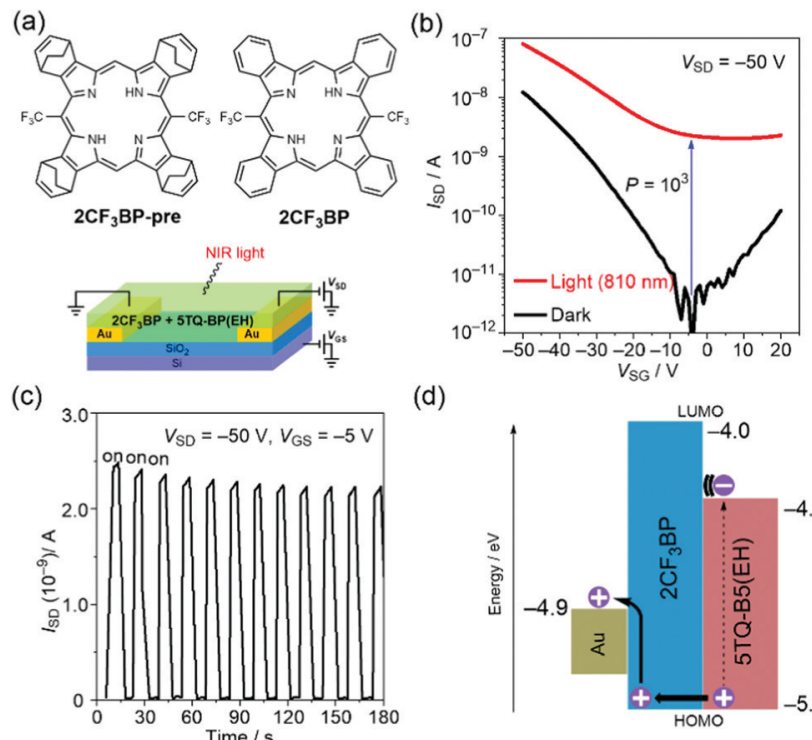

(d)

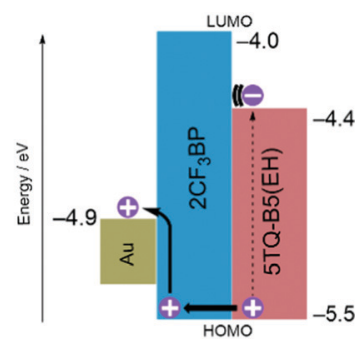

Fig. 9 (a) Chemical structures of $2 \mathrm{CF}_{3} \mathrm{BP}$-pre and $2 \mathrm{CF}_{3} \mathrm{BP}$ and OPT device architecture. (b) Transfer characteristics and (b) time-dependent switching response profile of the $\mathbf{5 T Q}-\mathbf{B} 5(\mathbf{E H}) / \mathbf{2} \mathrm{CF}_{3} \mathbf{B P}$-based NIR OPTs in the dark and under NIR light illumination. (d) Energy diagram of $2 \mathrm{CF}_{3} \mathbf{B P}$ and 5TQ-B5(EH).
The UV-Vis-NIR absorption spectrum of the 5TQ-B5(EH) film showed an absorption band in the NIR region with an absorption maximum at $806 \mathrm{~nm}$ (Fig. S10, ESI $\dagger$ ). Thus, we investigated the photoresponses of 5TQ-B5(EH) in the architecture of the OPTs (Fig. 9(a)). We adopted binary systems based on organic semiconductor and NIR sensitizer to construct the active layers of NIR photodetectors. ${ }^{26}$ The HOMO energy level of 5TQ-B5(EH) in thin film was determined to be $-5.5 \mathrm{eV}$ from the photoelectron spectroscopy measurements (Fig. S11, ESI $\dagger$ ). We chose a porphyrin derivative $\mathbf{2 C F}_{3} \mathbf{B P}$ as the counterpart of an organic semiconductor to reveal the OPT characteristics of 5TQ-B5(EH) as the NIR sensitizer in the binary system because $\mathbf{2 C F}_{3} \mathbf{B P}$ fulfills good energy matching of the HOMO level $(-5.5 \mathrm{eV})$ and weak absorbance at a wavelength of $810 \mathrm{~nm} \cdot{ }^{27}$ The bulk heterojunction configuration of the $\mathbf{5 T Q}-\mathbf{B 5}(\mathbf{E H}) / \mathbf{2 C F}_{3} \mathbf{B P}$ films were prepared by thermal conversion at $200{ }^{\circ} \mathrm{C}$ for $20 \mathrm{~min}$ in $\mathrm{N}_{2}$ atmosphere from the $\mathbf{5 T Q}-\mathbf{B}(\mathbf{E H}) / 2 \mathbf{C F}_{3} \mathbf{B P}-$ pre $(0.05 / 0.25 \mathrm{wt} \%)$ solutions to ascertain the area of semiconductor/NIR sensitizer interfaces. The OPT characteristics were measured in the dark and under NIR light illumination of $810 \mathrm{~nm}$ at an intensity of $143 \mathrm{~mW} \mathrm{~cm} \mathrm{~cm}^{-2}$ using light-emitting diodes. Fig. 9(b) shows that the transfer characteristics of the $\mathbf{5 T Q}-\mathbf{B 5}(\mathbf{E H}) / \mathbf{C F}_{3} \mathbf{B P}$-based OPTs under the bias voltage of the hole-transporting mode exhibits an apparent increase in the drain current $\left(I_{\mathrm{SD}}\right)$ when the active layer of the OPTs is exposed to NIR light. Photosensitivity $(P)$, as estimated from the equation $P=\left(I_{\text {light }}-I_{\mathrm{dark}}\right) / I_{\mathrm{dark}}$, is calculated to be $P=$ $10^{3}$. The specific detectivity $\left(D^{*}\right)$ of this device is calculated to be $3.8 \times 10^{8}$ Jones according to the equation $D^{*}=R \cdot S^{1 / 2} /\left(2 q I_{\mathrm{dark}}\right)^{1 / 2}$, where $R$ is the photoresponsivity, $S$ is the channel area, and $q$ is the electron charge. Fig. 9(c) shows that the time-dependent photoresponses were measured under NIR light on/off switching at $5 \mathrm{~s}$ intervals. The gate and drain voltages were set to -5 and $-50 \mathrm{~V}$, respectively. This device showed stable photoswitching behavior under repeated light on/off switching conditions. We considered that the hole generated by NIR light absorption of 5TQ-B5(EH) efficiently passes across the interface of 5TQ$\mathbf{B 5}(\mathbf{E H}) / \mathbf{2 C F}_{3} \mathbf{B P}$ and is collected at the drain electrode, which leads to the appearance of photosensitivity (Fig. 9(d)). ${ }^{28}$ These results indicate that $\pi$-extended quinoidal oligothiophenes with tuned closed-shell structures have the potential for use as an organic NIR-sensitive semiconducting material.

\section{Conclusions}

To control the quinoidal/aromatic contributions of long quinoidal oligothiophenes, a series of quinoidal pentathiophenes endcapped with dicyanomethylene units and bearing isothianaphthene units was designed. These molecules were successfully synthesized as stable structures by a thermal retro Diels-Alder reaction in the final step of the synthetic route. Theoretical calculations together with UV-Vis-NIR electronic absorption and vibrational FT-Raman spectroscopies showed that cross-conjugation between the annelating benzene moieties and the thiophene oligomer chain emerges upon increasing the number of benzene units. Concurrently, 
cross-conjugation imparts an increasing degree of the closedshell Kekule form, resulting in a new mode of tuning this conjugation in long quinoidal oligomers. More specifically, 5TQ-B(Ph) increased/decreased the contribution of quinoidal/ aromatic conjugation compared to 5TQ, whereas 5TQ-B5(Ph), which comprised fully of isothianaphthene units, exhibited significant cross-conjugation to further suppress the diradical character. Our elucidation of the competition between quinoid/ aromatic/cross-conjugation points to new structure-property relationships, which inspired us to fabricate OFETs based on the newly synthesized quinoidal pentathiophenes. These OFETs exhibited unipolar and ambipolar electrical semiconducting behaviors, which were further ascribed to the preferential stabilization of the reduced and/or oxidized species. Furthermore, 5TQ-B5(EH) exhibited NIR OPT characteristics that benefited from the relatively high current on/off ratio and NIR absorption behavior. Our results demonstrated that the combination of an extended quinoidal structure (providing NIR absorption) with isothianaphthene units (providing energy gap modulation and stability by cross-conjugation) is a promising design strategy for finetuning electronic structures in $\pi$-extended quinoidal oligothiophenes. Since we are able to further extend the molecular lengths of quinoidal oligothiophenes based on the results presented herein, the emergence of such strategies will help in the design of improved organic semiconductor materials that may pioneer novel electronic applications.

\section{Author contributions}

The manuscript was written through contributions of all authors.

\section{Conflicts of interest}

There are no conflicts to declare.

\section{Acknowledgements}

This work was supported by JSPS KAKENHI (20H02814, 20K21224, 20H05841, 20KK0123, 19K15505, 20H04639, 20K15352, 21K05213,20H00379, 20H05833, and 20K15261), CREST (J205101030), NEDO (21500248-0), and "Dynamic Alliance for Open Innovation Bridging Human, Environmental and Materials" from The Ministry of Education, Culture, Sports, Science and Technology, Japan. We are grateful to Prof. Toshihiro Ohnishi for helpful discussion. The authors thank the Spanish Ministry of Science, Innovation and Universities MCIU and MINECO/FEDER of the Spanish Government (project PGC2018-098533-B-100), the Ministry of Science and Technology of the Spanish Government (project RED2018102626-T) and the Junta de Andalucía, Spain (UMA18FEDERJA057). We also thank the Research Central Services (SCAI) of the University of Málaga.

\section{Notes and references}

1 J. Mei, Y. Diao, A. L. Appleton, L. Fang and Z. Bao, J. Am. Chem. Soc., 2013, 135, 6724-6746.

2 L. Lu, T. Zheng, Q. Wu, A. M. Schneider, D. Zhao and L. Yu, Chem. Rev., 2015, 115, 12666-12731.

3 G. Schweicher, G. Garbay, R. Jouclas, F. Vibert, F. Devaux and Y. H. Geerts, Adv. Mater., 2020, 32, 1905909.

4 L. Dou, Y. Liu, Z. Hong, G. Li and Y. Yang, Chem. Rev., 2015, 115, 12633-12665.

5 K. J. Baeg, M. Binda, D. Natali, M. Caironi and Y. Y. Noh, Adv. Mater., 2013, 25, 4267-4295.

6 S. R. González, Y. Ie, Y. Aso, J. T. López Navarrete and J. Casado, J. Am. Chem. Soc., 2011, 133, 16350-16353.

7 S. Handa, E. Miyazaki, K. Takimiya and Y. Kunugi, J. Am. Chem. Soc., 2007, 129, 11684-11685.

8 J. Casado, R. P. Ortiz and J. T. López Navarrete, Chem. Soc. Rev., 2012, 41, 5672-5686.

9 R. P. Ortiz, J. Casado, S. R. González, V. Hernández, J. T. López Navarrete, P. M. Viruela, E. Ortí, K. Takimiya and T. Otsubo, Chem. - Eur. J., 2010, 16, 470-484.

10 K. Yamamoto, S. I. Kato, H. Zajaczkowska, T. Marszalek, P. W. M. Blom and Y. Ie, J. Mater. Chem. C, 2020, 8, 3580-3588.

11 T. Mikie and I. Osaka, J. Mater. Chem. C, 2020, 8, 14262-14288.

12 C. Zhang, Y. Zang, E. Gann, C. R. McNeill, X. Zhu, C. A. Di and D. Zhu, J. Am. Chem. Soc., 2014, 136, 16176-16184.

13 (a) M. Abe, Chem. Rev., 2013, 113, 7011-7088; (b) J. J. Dressler, A. C. Valdivia, R. Kishi, G. E. Rudebusch, A. M. Ventura, B. E. Chastain, C. J. Gómez-Garcia, L. N. Zakharov, M. Nakano, J. Casado and M. M. Haley, Chem, 2020, 6, 1353-1368.

14 C. Zhang and X. Zhu, Acc. Chem. Res., 2017, 50, 1342-1350. 15 R. P. Ortiz, J. Casado, S. R. González, V. Hernández, J. T. López Navarrete, P. M. Viruela, E. Ortí, K. Takimiya and T. Otsubo, Chem. - Eur. J., 2010, 16, 470-484.

16 R. Ponce Ortiz, J. Casado, V. Hernandez, J. T. Lopez Navarrete, P. M. Viruela, E. Orti, K. Takimiya and T. Otsubo, Angew. Chem., Int. Ed., 2007, 46, 9057-9061.

17 T. Takahashi, K. I. Matsuoka, K. Takimiya, T. Otsubo and Y. Aso, J. Am. Chem. Soc., 2005, 127, 8928-8929.

18 (a) F. Wudl, M. Kobayashi and A. J. Heeger, J. Org. Chem., 1984, 49, 3382-3384; (b) J. L. Bredas, A. J. Heeger and F. Wudl, J. Chem. Phys., 1986, 85, 4673-4678; (c) Y. Ikenoue, F. Wudl and A. J. Heeger, Synth. Met., 1991, 40, 1-12; (d) H. Neugebauer, C. Kvarnström, C. Brabec, N. S. Sariciftci, R. Kiebooms, F. Wudl and S. Luzzati, J. Chem. Phys., 1999, 110, 12108-12115; (e) J. D. Douglas, G. Griffini, T. W. Holcombe, E. P. Young, O. P. Lee, M. S. Chen and J. M. J. Fréchet, Macromolecules, 2012, 45, 4069-4074; $(f)$ G. Long, X. Wan, J. Zhou, Y. Liu, Z. Li, G. He, M. Zhang, Y. Hou and Y. Chen, Macromol. Chem. Phys., 2012, 213, 1596-1603; $(g)$ W. A. Braunecker, Z. R. Owczarczyk, A. Garcia, N. Kopidakis, R. E. Larsen, S. R. Hammond, D. S. Ginley and D. C. Olson, Chem. Mater., 2012, 24, 1346-1356. 
19 (a) K. Yamamoto, Y. Ie, M. Nitani, N. Tohnai, F. Kakiuchi, K. Zhang, W. Pisula, K. Asadi, P. W. M. Blom and Y. Aso, J. Mater. Chem. C, 2018, 6, 7493-7500; (b) S. Moles Quintero, J. L. Zafra, K. Yamamoto, Y. Aso, Y. Ie and J. Casado, J. Mater. Chem. C, 2021, 9, 10727-10740.

20 K. Yamamoto, S. Jinnai, T. Takehara, T. Suzuki and Y. Ie, Org. Lett., 2020, 22, 547-551.

21 Y. Shimizu, Z. Shen, S. Ito, H. Uno, J. Daub and N. Ono, Tetrahedron Lett., 2002, 43, 8485-8488.

22 Handbook of Thiophene-Based Materials: Applications in Organic Electronics and Photonics, ed. I. F. Perepichka, D. F. Perepichka, John Wiley \& Sons, Ltd., Chichester, 2009.

23 (a) N. F. Phelan and M. Orchin, J. Chem. Educ., 1968, 45, 633-637; (b) R. Ponce, A. Facchetti, T. J. Marks, J. Casado, M. Z. Zgierski, M. Kozaki, V. Hernández and J. T. López Navarrete, Adv. Funct. Mater., 2009, 19, 386-394; (c) H. Hayashi, J. E. Barker, A. Cárdenas, R. Kishi, S. N. MacMillan, C. J. Gómez-García, H. Miyauchi, Y. Nakamura, M. Nakano, S. Kato, M. M. Haley and J. Casado, J. Am. Chem. Soc., 2020, 142, 20444-20455; (d) K. Kawabata, I. Osaka, M. Sawamoto, J. L. Zafra, P. Mayorga-Burrezo, J. Casado and K. Takimiya, Chem. Eur. J., 2017, 23, 4579-4589; (e) C. Zhang, S. M. Rivero, W. Liu, D. Casanova, X. Zhu and J. Casado, Angew. Chem.,
Int. Ed., 2019, 58, 11291-11295; $(f)$ K. Yang, X. Zhang, A. Harbuzaru, L. Wang, Y. Wang, C. Koh, H. Guo, Y. Shi, J. Chen, H. Sun, K. Feng, M. C. R. Delgado, H. Y. Woo, R. P. Ortiz and X. Guo, J. Am. Chem. Soc., 2020, 142, 4329-4340.

24 Z. Zeng, X. Shi, C. Chi, J. T. López Navarrete, J. Casado and J. Wu, Chem. Soc. Rev., 2015, 44, 6578-6596.

25 R. Kishi, S. Ochi, S. Izumi, A. Makino, T. Nagami, J.-y. Fujiyoshi, N. Matsushita, M. Saito and M. Nakano, Chem. - Eur. J., 2016, 22, 1493-1500.

26 (a) X. Ren, F. Yang, X. Gao, S. Cheng, X. Zhang, H. Dong and W. Hu, Adv. Energy Mater., 2018, 8, 1801003; (b) N. Li, Z. Lan, L. Cai and F. Zhu, J. Mater. Chem. C, 2019, 7, 3711-3729; (c) H. Xu, J. Li, B. H. K. Leung, C. C. Y. Poon, B. S. Ong, Y. Zhang and N. Zhao, Nanoscale, 2013, 5, 11850-11855; (d) S. Park, S. J. Kim, J. H. Nam, G. Pitner, T. H. Lee, A. L. Ayzner, H. Wang, S. W. Fong, M. Vosgueritchian, Y. J. Park, M. L. Brongersma and Z. Bao, Adv. Mater., 2015, 27, 759-765.

27 E. Jeong, K. Takahashi, S. K. Rajagopal, T. Koganezawa, H. Hayashi, N. Aratani, M. Suzuki, T. Q. Nguyen and H. Yamada, J. Org. Chem., 2020, 85, 168-178.

28 Y. Peng, W. Lv, B. Yao, G. Fan, D. Chen, P. Gao and M. Zhou, Org. Electron., 2013, 14, 1045-1051. 